\title{
ESTIGMA RELACIONADO A VIH/SIDA ASOCIADO CON ADHERENCIA AL TRATAMIENTO ANTIRRETROVIRAL EN PACIENTES DE UN HOSPITAL DE LIMA, PERÚ 2014
}

\author{
Jessica Hanae Zafra-Tanaka ${ }^{1, a}$, Eduardo Ticona-Chavez ${ }^{2, b}$
}

\begin{abstract}
RESUMEN
Objetivos. Determinar el nivel de estigma relacionado a VIH/SIDA y su asociación con la adherencia al tratamiento antirretroviral (TARV) en pacientes de un hospital nacional de Lima, Perú. Materiales y métodos. Estudio transversal que incluyó a pacientes adultos que seguían el programa de TARV con un mínimo de seis meses. Se consideró el estigma como la desvalorización de la persona por tener la infección de VIH y se midió con la escala de Berger para estigma relacionado a $\mathrm{VIH}$, la adherencia a la toma adecuada de los medicamentos fue medida con la encuesta Simplified medication adherence questionaire (SMAQ). Para evaluar los factores asociados a estigma se calcularon razones de prevalencia (RP) usando la regresión de Poisson. Resultados. Se analizaron 339 encuestas, la mediana de edad fue 39 años (RIC: 16 ) donde $74,0 \%$ eran hombres. Se halló un estigma alto en $25,4 \%$ y moderado en $47,5 \%$ de los encuestados. Una reducción de 10 puntos en la escala de estigma relacionado a VIH/SIDA se asoció a un aumento de $5 \%$ de adherencia al TARV en hombres (RPa 0,95, IC95\%: 0,91-0,99) y un aumento del 7\% en mujeres (RPa 0,93, IC95\%: 0,87-0,98). Conclusiones. Existe un alto nivel de estigma relacionado a VIH/SIDA y se asocia a la falta de adherencia al tratamiento con diferencias de acuerdo al sexo. Se recomiendan intervenciones dirigidas a reducir el estigma relacionado a divulgación de estatus en las mujeres y el relacionado a actitudes públicas en hombres.
\end{abstract}

Palabras clave: VIH; SIDA; Estigma social (fuente: DeCS BIREME).

\section{STIGMA RELATED TO HIV/AIDS ASSOCIATED WITH ADHERENCE TO ANTIRETROVIRAL THERAPY IN PATIENTS OF A PUBLIC HOSPITAL IN LIMA, PERU 2014}

\begin{abstract}
Objectives. To determine the level of stigma related to HIVIAIDS and its association with adherence to antiretroviral therapy (HAART). Materials and methods. A cross-sectional study that included adult patients which were part of the HAART program during at least 6 months. Stigma was defined as the loss of value of the person infected with HIV according to Berger scale and treatment adherence as the correct consume of medications which were measured with the Simplified Medication Adherence Questionnaire. Poisson regression test was used to calculate the prevalence ratios (PR) of associated factors. Results. The results of 339 surveys were analyzed. $74 \%$ were male and the median age was 39 years (Interquartile Range [IQR]: 16). High stigma was found in $25,4 \%$ of respondents, moderate in $47,5 \%$. A reduction of 10 points in the Berger scale was associated with an increase of $5 \%$ in adherence to HAART in men (adjusted PR [aPR]: 0.95; 95\% confidence interval [Cl]: 0.91-0.99), and 7\% in women (aPR: 0.92; 95\% Cl: 0.87-0.98). Conclusions. There is a high level of stigma related to HIVIAIDS and is associated with the lack of adherence to HAART according to gender. We recommend interventions aimed at reducing stigma related to status disclosure in women and related to public attitudes in men.
\end{abstract}

Keywords: HIV; AIDS; Social stigma (source: MeSH NLM)

\footnotetext{
Facultad de Medicina, Universidad Nacional Mayor de San Marcos. Lima, Perú.

Hospital Nacional Dos de Mayo. Lima, Perú

Médico cirujana; ${ }^{\mathrm{b}}$ médico especialista en enfermedades infecciosas y tropicales.

Este estudio forma parte de la tesis de Zafra-Tanaka, JH. Factores asociados al estigma relacionado al VIH/SIDA en los pacientes del programa TARGA del Hospital Nacional Dos de Mayo. Lima, Perú [Tesis para optar para el título de médico cirujano]. Lima: Facultad de Medicina, Universidad Nacional Mayor de San Marcos; 2015.

Recibido: 07/10/2015 Aprobado: 26/10/2016
}

Citar como: Zafra-Tanaka JH, Ticona-Chavez E. Estigma relacionado a VIH/SIDA asociado con adherencia al tratamiento antirretroviral en pacientes de un hospital de Lima, Perú 2014. Peru Med Exp Salud Publica. 2016;33(4): 625-32. doi: 10.17843/rpmesp.2016.334.2544 


\section{INTRODUCCIÓN}

La epidemia de VIH es un problema de salud pública, para el año 2008 en el Perú existían 76000 personas viviendo con VIH/SIDA (PVVS) de las cuales solo el $50 \%$ conocían su diagnóstico ${ }^{(1,2)}$. Durante el año 2013 se reportaron 938 casos de SIDA y 3005 casos de VIH nuevos. Según la Dirección General de Epidemiología del Perú, para noviembre de 2014 había 32491 casos diagnosticados de SIDA en el Perú y 55672 casos de $\mathrm{VIH}$, siendo así que aún existiría más de 20000 casos sin diagnosticar ${ }^{(3)}$.

El estigma relacionado con el VIH/SIDA es un proceso de desvalorización de las personas que viven con esta enfermedad o están asociadas con VIH/SIDA ${ }^{(4)}$. Debe entenderse que las personas afectadas por el estigma son tanto los infectados como los no infectados, y cada grupo posee manifestaciones diferentes ${ }^{(4,5)}$.

Según Earnshaw, los mecanismos que poseen las personas no infectadas son: el prejuicio, los estereotipos y la discriminación. Los prejuicios son las emociones y sentimientos negativos que tienen las personas no infectadas con respecto a las infectadas (ira, temor o disgusto). Los estereotipos son creencias de grupo sobre las PVVS, y la discriminación se refiere a la expresión de los prejuicios por medio de acciones. Esto puede dificultar la realización de la prueba de $\mathrm{VIH}$, apoyar a políticas sociales discriminatorias y de menor responsabilidad social y generar distanciamiento social ${ }^{(5)}$.

Los mecanismos que poseen las personas infectadas son: el percibido, el internalizado y el anticipado. El estigma percibido se refiere a cómo la PVVS siente que ha experimentado el prejuicio y la discriminación. La anticipación se refiere al grado en que las PVVS esperan ser víctimas de prejuicio o discriminación, y el estigma internalizado son los sentimientos y creencias negativas que poseen sobre sí mismas las PVVS. Estos se asocian con menor salud mental, menor soporte social y mayor cantidad de síntomas de $\mathrm{VIH}^{(5,6)}$.

El estigma afecta en mayor medida a ciertas poblaciones consideradas de riesgo, como hombres que tienen sexo con hombres $(\mathrm{HSH})$ y trabajadoras sexuales (TS) ${ }^{(7,8)}$. Esto se ve mediado por el abandono de la familia, el aislamiento social, la pérdida de empleo, la negación a los servicios de salud y la violencia ${ }^{(9)}$. Además, se ha encontrado que estos pueden influir en la demora al acudir a los servicios de salud y realizar una prueba de $\mathrm{VIH}$, la revelación del estado a la pareja (lo que a su vez perpetúa la propagación de la enfermedad), y la adherencia terapéutica ${ }^{(9-12)}$. Así, el estigma relacionado a VIH constituye un impedimento para el acceso a la prevención, diagnóstico y tratamiento del VIH/SIDA.
La adherencia al tratamiento, entendida como el contacto entre el usuario y los servicios de salud o toma del 90 a $95 \%$ de las dosis, ${ }^{(13)}$ es influenciada por el estigma de manera tal que, a mayor estigma relacionado a VIH se presentará menor adherencia y riesgo de abandono del tratamiento. ${ }^{(14-16)}$

El objetivo del presente estudio fue determinar el nivel de estigma relacionada a VIH/SIDA e identificar su asociación con la adherencia al tratamiento antirretroviral (TARV) en pacientes del programa de uno de los hospitales públicos que brindan gran cobertura a PVVS en Perú.

\section{MATERIALES Y MÉTODOS}

\section{DISEÑO Y POBLACIÓN DE ESTUDIO}

Se realizó un estudio observacional transversal analítico. La población estuvo compuesta por pacientes con $\mathrm{VIH} /$ SIDA dentro del programa TARV del Hospital Nacional Dos de Mayo en Lima, Perú durante el año 2014. Se incluyó a personas $\geq 18$ años, con un mínimo de seis meses dentro del programa de TARV y siguiéndolo al momento de la recolección de los datos. Se excluyó a los pacientes con incapacidad física o mental para responder la encuesta y aquellos que no brindaron su consentimiento informado.

El tamaño de muestra se determinó con el programa Epidat 3.1 con un nivel de confianza de 95\%, una proporción esperada de estigma relacionado a $\mathrm{VIH} /$ SIDA de $60 \%$ y una precisión de $5 \%$. Sobre la base de una población estimada de 2000 personas, según lo reportado por los médicos al Servicio de Infectología de este hospital, se calculó un tamaño muestral de 312 personas.

El muestreo fue por conveniencia ya que para mantener la confidencialidad de los pacientes no era posible obtener una lista y utilizarla de marco muestral, por lo tanto, se encuestó a los pacientes que acudieron a sus controles entre los meses de octubre a diciembre de 2014 , teniendo en cuenta que los controles se realizan cada tres meses.

\section{INSTRUMENTO DE RECOLECCIÓN DE DATOS}

Se aplicó una encuesta autoadministrada que contaba con tres secciones, la primera describía a las variables sociodemográficas; la segunda, la adherencia al tratamiento TARV para lo cual se utilizó la encuesta Simplified medication adherence questionaire (SMAQ) ${ }^{(17)}$, y la última parte estuvo conformada por la escala Berger que mide estigma relacionado a $\mathrm{VIH} / \mathrm{SIDA}$, previamente validada en Perú (12). 


\section{MEDICIÓN DE ESTIGMA RELACIONADO A VIH/SIDA}

El estigma fue evaluado con la escala Berger, la cual cuenta con 21 preguntas divididas en cuatro dimensiones: estigma establecido (cinco peguntas), preocupaciones de divulgación de estatus (cinco peguntas), autoimagen negativa (seis peguntas) y preocupaciones con actitudes públicas (cinco peguntas). Las alternativas están en escala de Likert (casi nunca, rara vez, a veces y casi siempre), y otorgan de uno a cuatro puntos por ítem. Finalmente, la suma de puntajes permite obtener un puntaje total mínimo de 21 y máximo de 84 puntos ${ }^{(18)}$. Los valores finales utilizados fueron, mínimo a moderado (21-61) y alto (62-84). Esta encuesta fue validada en Perú y cuenta con una fiabilidad determinada con el coeficiente alfa de Cronbach de $0,89^{(12)}$.

\section{MEDICIÓN DE ADHERENCIA AL TRATAMIENTO}

La encuesta SMAQ tiene seis preguntas con respuesta cerrada. Se consideró como no adherente a aquellas personas que tuvieron una respuesta positiva a alguna de las preguntas (Tabla 1). Para las útlimas dos preguntas, se consideró como positivo el olvido de tomar más de dos dosis en la úlima semana o el olvido de tomar el medicamento por más de 2 días en los últimos 3 meses. Esta encuesta fue validada en otro estudio donde se halló una sensibilidad de $72 \%$, especificidad de $91 \%$ y fiabilidad determinada con el coeficiente alfa de Cronbach de 0,75 (17). Además, ha sido utilizada dentro del programa de TARV en el hospital Loayza (13).

Como parte de la validación de apariencia, tres expertos en el tema (un médico epidemiólogo especialista en infecciones de transmisión sexual y $\mathrm{VIH}$, un médico salubrista y un infectólogo) evaluaron la encuesta de forma virtual y luego de las modificaciones indicadas se obtuvo un índice de concordancia de $93 \%$, con lo que se procedió a aplicar la encuesta.

\section{RECOLECCIÓN DE DATOS}

La encuesta se realizó en las instalaciones del programa TARV del Hospital Nacional Dos de Mayo. Los pacientes fueron abordados por los consejeros de pares mientras esperaban su atención ambulatoria. Los consejeros de pares recibieron una capacitación que consistió en la explicación de los objetivos del estudio y una revisión de todos los ítems de la encuesta para que puedan resolver las dudas de los encuestados.

Se obtuvo el consentimiento informado firmado luego de una breve explicación de los objetivos del estudio y las características de las encuesta. Aquellos que aceptaban participar del proyecto llenaron las encuestas en un ambiente privado en la sala de espera del programa.

\section{ANÁLISIS ESTADÍSTICO}

Se analizaron las variables categóricas por medio de frecuencias absolutas y relativas. Las variables cuantitativas que no presentaron distribución normal, según la prueba de Kolmorogov-Smirnov, se resumieron en mediana y rangos.

Se calculó la razón de prevalencias (RP) con un intervalo de confianza del 95\% (IC95\%) como medida de fuerza de asociación mediante el modelo de regresión de Poisson con varianza robusta. Esta prueba fue utilizada también para el análisis multivariado en el que se incluyeron aquellas variables que se asociaron de forma significativa en el análisis bivariado $(p<0,05)$ y aquellas consideradas asociadas al estigma según la bibliografía consultada ${ }^{(4,10,19)}$. Para el análisis de la relación entre el estigma y la adherencia al tratamiento se utilizó el puntaje total obtenido de la escala Berger para estigma y de sus dimensiones por separado. El análisis se realizó con el programa STATA $v 12$.

\section{CONSIDERACIONES ÉTICAS}

La evaluación ética del estudio fue realizada por los comités de ética en investigación de la Facultad de Medicina de la Universidad Nacional Mayor de San Marcos (Lima, Perú) y del Hospital Nacional Dos de Mayo (Lima, Perú). La información brindada por los encuestados fue confidencial y anónima, ya que no se registró el nombre de los participantes en las encuestas

Tabla 1. Calificación de la adherencia al tratamiento antirretroviral según cuestionario SMAQ

\begin{tabular}{lcc}
\hline \multicolumn{1}{c}{ Cuestionario Simplified Medication Adherence Questionaire (SMAQ) } & Sí & (\%) \\
\hline 1. ¿Alguna vez olvida tomar la medicación? & 186 & $(54,9)$ \\
2. ¿Tomó siempre los medicamentos a la hora indicada? & 265 & $(78,4)$ \\
3. ¿Alguna vez deja de tomar las medicinas si se siente mal? & 44 & $(13,0)$ \\
4. ¿Olvidó tomar la medicación durante el fin de semana? & 55 & $(16,3)$ \\
5. No tomó más de dos dosis en la última semana & 7 & $(2,1)$ \\
6. ¿Olvidó tomar el medicamento más de dos días, en los últimos 3 meses? & 44 & $(13,0)$ \\
No adherentes * & 226 & $(66,7)$ \\
\hline
\end{tabular}

* Pacientes que han contestado afirmativamente alguna de las siguientes preguntas: 1, 3, 4, 5, o 6; o que han contestado negativamente la pregunta 2 
y el consentimiento informado no tenía un código de identificación que permitiera relacionar las respuestas de la encuesta con la identidad de la persona. Cada persona decidió libremente participar en el estudio y de abstenerse de responder alguna pregunta incómoda.

\section{RESULTADOS}

Fueron encuestadas 466 personas, 43 de ellas no cumplieron con los criterios de inclusión por tener menos de seis meses en el programa TARV, y 84 encuestas fueron anuladas debido a falta de datos o inconsistencia entre los datos obtenidos. Finalmente, se analizaron 339 encuestas. Los resultados de la escala de Berger para estigma relacionado a VIH/SIDA se presenta en la Figura 1.

La mediana de la edad fue de 39 años (RIC: 16); 251 $(74,0 \%)$ fueron hombres; $55(16,2 \%)$ personas no poseía domicilio fijo; 147 (44,7\%) estaban desempleados; 108 (31,95\%) estaban unidos, y 47,2\% (160) poseía estudios superiores. Con respecto a la orientación sexual, 197 $(59,2 \%)$ se consideraron heterosexuales; $154(45,7 \%)$ poseían pareja estable, de estos, $23(15,2 \%)$ no habían revelado su estado seropositivo a su pareja.

La mediana del tiempo viviendo con diagnóstico de VIH/ SIDA fue de 60 meses (RIC: 72 meses), mientras la mediana del tiempo dentro del programa TARV fue de 48 meses (RIC: 60 meses).
De los encuestados, 86 (25,4\%) presentaron estigma relacionado a $\mathrm{VIH} /$ SIDA alto, y la media para el puntaje total de la escala de Berger fue de 53,7 (DE=12,02). Para las dimensiones de estigma relacionado a $\mathrm{VIH} /$ SIDA, se halló una media de 10,47 puntos (DE= $4,46)$ para estigma establecido; $16,22(\mathrm{DE}=3,84)$ para preocupación de divulgación de estatus; 9,91 (DE=7,09) para autoimagen negativa y 15,63 (DE=3,78) para preocupaciones con actitudes públicas (Figura 1).

De las personas encuestadas, el $66,7 \%$ (226) no tuvo adherencia al tratamiento TARV. Las personas encuestadas tomaban una mediana de tres pastillas al día (RIC: 3) (Tabla 1).

En el análisis bivariado, personas con educación secundaria presentaron $61 \%$ menos estigma alto (RP 0,39, IC95\%: 0,24-0,62) y 54\% menos estigma si poseían educación superior (RP 0,46, IC95\% 0,30-0,73). Tener domicilio fijo se asoció con $60 \%$ menos estigma alto (RP: 0,40 IC95\%: 0,28-0,57) y el tener un empleo con un $34 \%$ menos de estigma alto (RP 0,66, IC95\%: $0,46-0,97)$. En el análisis multivariado, se halló que aquellas personas con educación secundaria presentan $58 \%$ menos estigma alto (RP 0,42 IC95\% 0,25-0,72) y personas con domicilio fijo presentaron $59 \%$ menos estigma alto (RP 0,41 IC95\%: 0,28-0,59) (Tabla 2).

En el análisis entre estigma y adherencia al tratamiento ajustado por edad, tiempo en TARV, estado civil, grado

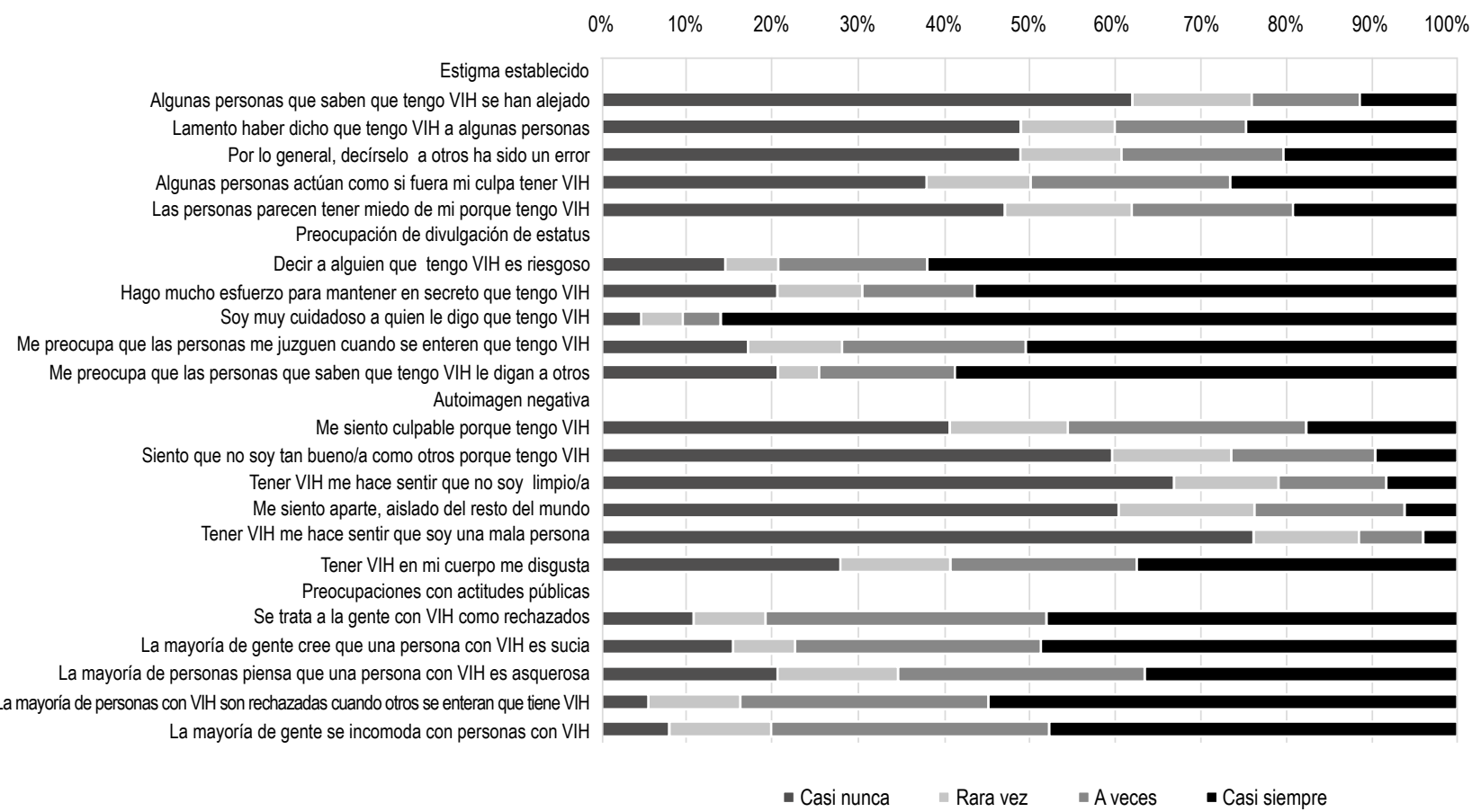

Figura 1. Estigma relacionado a VIH/SIDA según escala de Berger en pacientes del programa de tratamiento antirretroviral del Hospital Nacional Dos de Mayo, Lima Perú 2014 ( $n=339)$ 
Tabla 2. Factores asociados a estigma relacionado a VIH/SIDA en pacientes del programa de tratamiento antirretroviral del Hospital Nacional Dos de Mayo. Lima, Perú 2014

\begin{tabular}{|c|c|c|c|c|c|}
\hline & & $\begin{array}{c}\text { Estigma mínimo } \\
\text { a moderado }\end{array}$ & Estigma alto & & \\
\hline & $\mathbf{N}$ & $\mathrm{n}=253(\%)$ & $n=86(\%)$ & $\mathrm{RP}^{*}$ (IC 95\%) & $\mathrm{RPa}^{* *}$ (IC 95\%) \\
\hline \multicolumn{6}{|l|}{ Sexo } \\
\hline Femenino & 88 & $65(73,9)$ & $23(26,1)$ & 1,00 & 1,00 \\
\hline Masculino & 251 & $188(74,9)$ & $63(25,1)$ & $0,96(0,63-1,45)$ & $1,01(0,63-1,61)$ \\
\hline \multicolumn{6}{|l|}{ Edad } \\
\hline 19 a 35 años & 127 & $90(70,9)$ & $37(29,1)$ & 1,00 & 1,00 \\
\hline 36 a 44 años & 103 & $85(82,5)$ & $18(17,5)$ & $0,59(0,36-0,99)$ & $0,75(0,45-1,23)$ \\
\hline 45 a 76 años & 103 & $74(71,8)$ & $29(28,2)$ & $0,97(0,64-1,45)$ & $0,96(0,60-1,52)$ \\
\hline \multicolumn{6}{|l|}{ Grado de instrucción } \\
\hline Primaria & 26 & $12(46,2)$ & $14(53,8)$ & 1,00 & 1,00 \\
\hline Secundaria & 153 & $121(79,1)$ & $32(20,9)$ & $0,39(0,24-0,62)$ & $0,42(0,25-0,72)$ \\
\hline Superior & 160 & $120(75,0)$ & $40(25,0)$ & $0,46(0,30-0,73)$ & $0,64(0,36-1,14)$ \\
\hline \multicolumn{6}{|l|}{ Estado civil } \\
\hline Unido & 108 & $77(71,3)$ & $31(28,7)$ & 1,00 & 1,00 \\
\hline No unido & 230 & $175(76,1)$ & $55(23,9)$ & $0,83(0,57-1,21)$ & $0,73(0,49-1,09)$ \\
\hline \multicolumn{6}{|l|}{ Domicilio fijo } \\
\hline No & 55 & $27(49,1)$ & $28(50,9)$ & 1,00 & 1,00 \\
\hline Sí & 284 & $226(79,6)$ & $58(20,4)$ & $0,40(0,28-0,57)$ & $0,41(0,28-0,59)$ \\
\hline \multicolumn{6}{|l|}{ Empleo } \\
\hline No & 147 & $101(68,7)$ & $46(31,3)$ & 1,00 & 1,00 \\
\hline Sí & 182 & $144(79,1)$ & $38(20,9)$ & $0,66(0,46-0,97)$ & $0,71(0,47-1,08)$ \\
\hline \multicolumn{6}{|l|}{ Orientación sexual } \\
\hline Heterosexual & 197 & $144(73,1)$ & $53(26,9)$ & 1,00 & 1,00 \\
\hline No heterosexual & 136 & $106(77,9)$ & $30(22,1)$ & $0,82(0,55-1,21)$ & $0,92(0,56-1,52)$ \\
\hline \multicolumn{6}{|c|}{ Tiempo viviendo con VIH } \\
\hline $6-48$ meses & 140 & $104(74,3)$ & $36(25,7)$ & 1,00 & 1,00 \\
\hline 49 - 96 meses & 101 & $69(68,3)$ & $32(31,7)$ & $1,23(0,82-1,84)$ & $1,10(0,72-1,67)$ \\
\hline $97-276$ meses & 98 & $80(81,6)$ & $18(18,4)$ & $0,71(0,43-1,18)$ & $0,84(0,49-1,44)$ \\
\hline
\end{tabular}

${ }^{*}$ Razón de prevalencia, **azón de prevalencia justada

de instrucción y orientación sexual, se halló que en los hombres una reducción de 10 puntos en la escala de estigma relacionado a $\mathrm{VIH} / \mathrm{SIDA}$ se asocia a $5 \%$ más de adherencia al TARV (RPa 0,95, IC95\%: 0,91-0,99); para las mujeres, esta reducción se asocia a un aumento de 7\% en la adherencia al tratamiento (RPa 0,93, IC95\%: 0,870,98). Según las dimensiones de la escala de estigma relacionado a $\mathrm{VIH} / \mathrm{SIDA}$ en los hombres, una reducción de 10 puntos en la dimensión de preocupación sobre actitudes públicas se asocia a un aumento de $14 \%$ de adherencia (RPa 0,86, IC95\%: 0,76-0,96). En las mujeres, una reducción de 10 puntos en la preocupación de divulgación de estatus se asocia a $20 \%$ más adherencia al tratamiento (RPa 0,80, IC95\%: 0,68-0,95) Tabla 3.

Tabla 3. Asociación entre estigma relacionado a VIH/SIDA y adherencia al tratamiento antirretroviral en pacientes del Hospital Nacional Dos de Mayo. Lima 2014

\begin{tabular}{|c|c|c|c|}
\hline & \multicolumn{3}{|c|}{ Adherencia al tratamiento antirretroviral } \\
\hline & Total & Hombres & Mujeres \\
\hline & $\mathrm{RPa}^{*}$ (IC 95\%) & $\mathrm{RPa}^{*}$ (IC 95\%) & $\mathrm{RPa}^{*}$ (IC 95\%) \\
\hline Estigma relacionado a VIH/SIDA & $0,94(0,91-0,98)^{\star \star}$ & $0,95(0,91-0,99)^{\star \star}$ & $0,93(0,87-0,98)^{\star \star}$ \\
\hline \multicolumn{4}{|c|}{$\begin{array}{l}\text { Dimensiones de la escala de estigma relacionado a } \mathrm{VIH} / \\
\text { SIDA }\end{array}$} \\
\hline Estigma establecido & $0,91(0,83-0,99)^{* *}$ & $0,90(0,81-1,00)^{* *}$ & $0,95(0,83-1,09)$ \\
\hline Preocupación de divulgación de estatus & $0,91(0,82-1,00)$ & $0,91(0,81-1,03)$ & $0,80(0,68-0,95)^{\star *}$ \\
\hline Autoimagen negativa & $1,03(0,98-1,09)$ & $1,02(0,95-1,09)$ & $1,08(0,98-1,19)$ \\
\hline Preocupaciones con actitudes públicas & $0,87(0,78-0,97)^{\star *}$ & $0,86(0,76-0,96)$ & $0,84(0,66-1,07)$ \\
\hline
\end{tabular}

* ajustado por edad, grado de instrucción, orientación sexual, estado civil, domicilio fijo, empleo y tiempo dentro del programa. ${ }^{* *} p<0,05$ 


\section{DISCUSIÓN}

La cuarta parte de los pacientes poseen estigma relacionado a VIH/SIDA alto. Este se asocia a niveles de educación inferior y a falta de domicilio fijo. Además, el estigma se asocia a la adherencia al tratamiento de distinta forma en hombres y mujeres.

En el presente estudio la media del puntaje total de la escala Berger fue mayor que lo reportado en otro estudio realizado en Perú en grupos de tratamiento directamente observado administrado en casa, donde se hallaron medias de $46(D E=16)$ y $51(D E=14)$ en los grupos estudiados ${ }^{(11)}$. En nuestro estudio se halló que las dimensiones más afectadas fueron la preocupación de divulgación de estatus y las actitudes públicas, al igual que en un estudio realizado en Lima, en el cual estas dimensiones se asociaron a falta de retención en los cuidados de $\mathrm{VIH}{ }^{(16)}$.

La inclusión de pacientes luego de seis meses de TARV puede haber disminuido la magnitud del estigma en los resultados obtenidos, ya que se ha hallado que este disminuye con el tiempo viviendo con el diagnóstico. Así, luego de 12 meses de TARV se ha evidenciado una disminución del estigma, inclusive en grupos donde no se realizó una intervención para controlarlo ${ }^{(11,18,19)}$. Se recomienda realizar estudios longitudinales que permitan evaluar la variación del estigma a través del tiempo y qué implicancias puede tener tanto en la adherencia al tratamiento como en la calidad de vida de las personas dentro del programa ${ }^{(15)}$.

Dentro del programa TARV, aquellas personas con falta de un domicilio fijo y solo con educación primaria, presentaron mayor frecuencia de estigma alto. La falta de domicilio fijo puede ser reflejo de inadecuados ingresos económicos que interactúan con el estigma de dos maneras: la enfermedad disminuye la capacidad laboral de las personas y esto lleva a la exclusión o el estigma hace que la persona se aislé y le impida conseguir refugio ${ }^{(14)}$. Se recomienda abordar a los pacientes con estas características y brindarles consejería que pueda disminuir el estigma relacionado a VIH.

La epidemia de VIH en el Perú es de tipo concentrada, por lo cual la población de $\mathrm{HSH}^{(20)}$ es más afectada. En un estudio realizado en un hospital de la ciudad de Ica en Perú, que evaluó pacientes en TARV, se halló que el $12 \%$ eran homosexuales y $8 \%$ bisexuales ${ }^{(21)}$ a diferencia del nuestro donde se halló 31,9 y $7,7 \%$, respectivamente, lo que puede deberse a la encuesta ofrecida con consejeros de pares, autoadministrada y confidencial, que brinda mayor seguridad para revelar la orientación sexual. Si bien no se halló relación entre el estigma y la orientación sexual, se recomienda realizar estudios en esta población y brindar canales para la obtención de apoyo dentro de la comunidad LGTB (lesbianas, gais, transexuales y bisexuales) ya que esto puede ayudar a disminuir el estigma. Además, se debe prestar atención en aquellas personas que mantienen relaciones sexuales con personas del mismo sexo, pero no se consideran homosexuales, ya que la falta de identificación y apoyo de grupo se ha asociado a mayor estigma en estas personas ${ }^{(22)}$.

La no adherencia al TARV se halló en $66,7 \%$, mientras que en un estudio realizado en otro hospital en Lima fue de $35,9 \%$ para el año $2009{ }^{(13)}$. Lo que podría deberse a que dicho hospital incluyó pacientes desde el primer mes en TARV, donde, en teoría, hay mejor adherencia, a diferencia del enrolamiento realizado en nuestro estudio a partir del sexto mes. En adición, esa población tuvo menor tiempo en TARV (media de 15,6 meses ( $D E=10,9)$ vs $55,7(\mathrm{DE}=38) \mathrm{y}$, por ende, mayor probabilidad de mejor adherencia ${ }^{(15)}$. A pesar de ello, la meta de $90 \%$ de adherencia al tratamiento TARV se encuentra lejos ${ }^{(23)}$.

El sexo y la orientación sexual influyen en la adherencia al tratamiento, ya que condicionan formas de aproximación a los servicios de salud distintos ${ }^{(24)}$. En otro estudio que se llevó a cabo en un hospital de Lima se asoció también el tener una orientación distinta de la heterosexual, no tener domicilio fijo y el mayor tiempo en el tratamiento ${ }^{(13)}$. Sin embargo, no se halló esta relación en nuestro estudio.

El estigma constituye un fenómeno importante que afecta no solo la adherencia, sino que también se relaciona con el abandono del tratamiento ${ }^{(16)}$. Los mecanismos mediante los cuales el estigma afecta la adherencia han sido descritos a diversos niveles; individual, grupal y general. Hemos hallado que el estigma total afecta de forma negativa la adherencia en hombres y mujeres; sin embargo, diversas dimensiones del estigma pueden afectar a estos grupos por mecanismos diferentes. En general, en base a estudios realizados en su mayoría en hombres, se acepta que el estigma percibido afecta al internalizado y este, a su vez, se asocia a la negación de la enfermedad o a la falta de revelación de estado a la pareja o familiares, lo que lleva a ausencia de apoyo social y aislamiento ${ }^{(14,25,26)}$. Las estrategias de afrontamiento negativas se perpetúan por el estigma, e impactan de forma negativa en la adherencia al tratamiento. Debido a que esta constituye un pilar importante en la cascada del continuo de atención del $\mathrm{VIH}{ }^{(23)}$ se recomienda realizar intervenciones sobre el estigma para mejorar la adherencia y hacer el seguimiento con evaluaciones periódicas utilizando una encuesta estandarizada para obtener datos cuantitativos que permitan contrastar resultados anteriores. 
Si bien el género no se encontró asociado a mayores niveles de estigma, hay estudios donde se ha hallado que las mujeres presentaban mayor riesgo de presentar estigma elevado y que ante situaciones de discriminación similares las mujeres son más propensas a internalizar el estigma (19,27,28). En nuestro medio, la condición de VIH/SIDA se relaciona a conductas "inmorales"; así, las mujeres presentan estigma percibido proveniente de amigos, familia y personal de salud (médicos y enfermeras) que no favorece el contacto posterior con los servicios de salud ${ }^{(29)}$. Otros mecanismos que median la relación entre estigma y adherencia en mujeres son: el aislamiento, la falta de soporte social y los síntomas depresivos, por lo que se han sugerido estrategias como fortalecer la terapia en salud mental y el desarrollo de habilidades sociales (26). En nuestra población, recomendamos enfatizar en las preocupaciones sobre la divulgación de estatus, ya que una mejora de 10 puntos, según la escala de Berger, en esta dimensión, generaría un aumento de la adherencia al tratamiento de $20 \%$. Cabe resaltar que el estigma condiciona que se prolongue la revelación del diagnóstico, sobre todo en personas con relaciones estables de larga duración basadas en la confianza que representan una fuente importante de apoyo social ${ }^{(22)}$.

En el caso de los varones, se recomienda trabajar en las preocupaciones por actitudes públicas, ya que una mejora en esta dimensión del estigma medida por la escala de Berger, puede ser traducida en $14 \%$ más adherencia.

Las limitaciones que se tuvieron fueron: el muestreo por conveniencia, que puede ocasionar un sesgo de selección. ya que se podría argumentar que aquellas personas que aceptaron realizar la encuesta presentaban menor estigma que aquellas que no aceptaron. Esto se trató de minimizar contando con el apoyo de los consejeros de pares del programa TARV, con quienes tienen una relación de confianza y apoyo, a través de los cuales se invitó a los participantes. Además, al tratarse de un estudio transversal no es posible determinar una relación de causalidad entre el estigma y la adherencia debido a la temporalidad.

En el presente estudio se abordó el estigma individual de la persona viviendo con $\mathrm{VIH} / \mathrm{SIDA}$, sin embargo, este proceso de desvalorización posee también un componente superior al individual como es el estructural. Este se encuentra en relación a la forma en la cual diversas políticas del estado o del hospital pueden generar discriminación en este grupo de personas (30). De esta manera los carteles de señalización localizados en el área de atención de estos pacientes con título de "PROCETSS" o "consultorios TARGA" podrían representar una marca. Además, diversos aspectos sociales y de salud mental se encuentran presentes en las personas viviendo con VIH/SIDA que no han sido evaluados en este estudio como la depresión o el pobre apoyo social ${ }^{(11,15,18)}$.

Se concluye que la cuarta parte de los pacientes poseen estigma relacionado a VIH/SIDA alto. Este se asocia con la adherencia al tratamiento antirretroviral de distinta forma en hombres y mujeres, por este motivo, se recomienda realizar intervenciones para mejorar el estigma en relación a divulgación de estatus en las mujeres y el relacionado con actitudes públicas en el caso de varones.

Contribución de los autores: JHZT participó de la concepción y diseño del artículo, recolección y obtención de resultados y de la obtención de financiamiento. Ambos autores participaron del análisis e interpretación de datos, redacción de artículo, revisión crítica y aprobación de la versión final. En adición el autor ETC participó en el aporte de pacientes y material de estudio.

Fuentes de financiamiento: se obtuvo financiamiento mediante un concurso organizado por la Sociedad Científica Médico Estudiantil Peruana (SOCIMEP).

Conflictos de interés: los autores declaran no tener conflictos de interés en la publicación de este artículo.

\section{REFERENCIAS BIBLIOGRÁFICAS}

1. USAID. HIV/AIDS health profile: Perú. Septiembre 2011. [CItado el 01/02/2015] Disponible en: http:// www.usaid.gov/our_work/global_ health/aids/Countries/lac/peru_ profile.pdf

2. WHO, UNICEF, UNAIDS. Progress Report 2011: Global HIV/AIDS response. Epidemic update and health sector progress towards Universal Access . Malta, 2011.
3. Dirección General de Epidemiología. Boletín epidemiológico mensual: Situación del VIH/SIDA en el Perú (Nov 2014). [Citado el 08/10/16] Disponible en: http://www.dge.gob. pe/portal/

4. ONUSIDA. Hoja informativa del ONUSIDA sobre el estigma y la discriminación. 2003.

5. Earnshaw VA, Chaudoir SR. From conceptualizing to measuring HIV stigma: a review of HIV stigma mechanism measures. AIDS Behav. 2009;13(6):1160-77.

6. Pichon LC, Rossi KR, Ogg SA, Krull LJ, Young-Griffin D. Social Support, Stigma and Disclosure: Examining the Relationship with HIV Medication Adherence among Ryan White Program Clients in the Mid-South USA. Int J Environ Res Public Health. 2015;12(6):7073-84. 
7. ONUSIDA. Reducir el estigma y la discriminación por el VIH: una parte fundamental de los programas nacionales del sida. Ginebra: Programa Conjunto de las Naciones Unidas sobre el VIH/Sida. 2008.

8. Cáceres CF, Mendoza W. The National Response to the HVI/AIDS Epidemic in Peru: Accomplishments and Gaps- A Review. J Acquir Immune Defic Syndr. 2009;51(Supplement 1):S60-S6.

9. Do NT, Phiri K, Bussmann $\mathrm{H}$, Gaolathe T, Marlink RG, Wester CW. Psychosocial factors affecting medication adherence among HIV-1 infected adults receiving combination antiretroviral therapy (cART) in Botswana. AIDS Res Hum Retroviruses. 2010;26(6):685-91.

10. Gonzalez A, Miller CT, Solomon SE, Bunn JY, Cassidy DG. Size matters: community size, HIV stigma, \& gender differences. AIDS Behav. 2009;13(6):1205-12.

11. Munoz M, Finnegan K, Zeladita J, Caldas A, Sanchez E, Callacna $\mathrm{M}$, et al. Community-based DOTHAART accompaniment in an urban resource-poor setting. AIDS Behav. 2010;14(3):721-30.

12. Franke MF, Finnegan K, Zeladita J, Sebastian JL, Bayona JN, Shin. SS. Validation and Abbreviation of an HIV Stigma Scale in an Adult SpanishSpeaking Population in Urban Peru. AIDS Behav. 2010;14(1):189-99.

13. Alvis Ó, L De Coll, Chumbimune L, Díaz C, Díaz J, Reyes M. Factores asociados a la no adherencia al tratamiento antirretroviral de gran actividad en adultos infectados con el VIH-sida. An Fac med. 2009;70(4).

14. Katz IT, Ryu AE, Onuegbu AG, Psaros C, Weiser SD, Bangsberg DR, et al. Impact of HIV-related stigma on treatment adherence: systematic review and meta-synthesis. J Int AIDS Soc. 2013;16(3Suppl 2).

15. Sayles JN, Wong MD, Kinsler JJ, Martins D, Cunningham WE. The Association of Stigma with SelfReported Access to Medical Care and Antiretroviral Therapy Adherence in Persons Living with HIV/AIDS. J Gen
Intern Med. 2009;24(10):1101-8.

16. Valenzuela C, Ugarte-Gil C, Paz J, Echevarría J, Gotuzzo E, Vermund SH, et al. HIV Stigma as a barrier to retention in HIV care at a general hospital in Lima, Peru: a casecontrol study. AIDS Behav. 2015 Feb;19(2):235-45. doi: 10.1007/ s10461-014-0908-7.

17. Knobel H, Alonso J, Casado J, Collazos J, Gonzalez J, Ruiz I, et al. Validation of a simplified medication adherence questionnaire in a large cohort of HIVinfected patients: the GEEMA Study. AIDS. 2002;16(4):605-13.

18. Kaai S, Bullock S, Sarna A, Chersich M, Luchters S, Geibel S, et al. Perceived stigma among patients receiving antiretroviral treatment: a prospective randomised trial comparing an m-DOT strategy with standard-of-care in Kenya. SAHARA J. 2010;7(2):6270.

19. Loutfy MR, Logie $\mathrm{CH}$, Zhang $\mathrm{Y}$, Blitz SL, Margolese SL, Tharao WE, et al. Gender and Ethnicity Differences in HIV-related Stigma Experienced by People Living with HIV in Ontario, Canada. PLoS ONE. 2012;7(12):e48168.

20. Ministerio de Salud - Perú. Informe Nacional sobre los progresos realizados en el país (Periodo enero 2010 y diciembre 2011). 2012.

21. Bonilla-Ruiz AM, VilcapomaBalbín P. Características clínicas, epidemiológicas, inmunoserológicas de los pacientes en terapia antiretroviral de gran actividad de un hospital peruano. Rev Med panacea. 2013;3(1):19-23.

22. Flores F, Almanza M. Un estudio exploratorio de las consecuencias psicosociales del estigma en varones que viven con VIH en México. Revista Iberoamericana de Psicología: Ciencia y Tecnología. 2013;66(1):51-9.

23. WHO/PAHO. Continuum of care Monitoring Framework. Addendum to meeting report: Regional consultation on HIV apidemiologic information in Latin America and the Caribbean. 2014.

24. Stuardo-Ávila V, Manriquez-Urbina JM, Fajreldin-Chuaqui V, Belmar-
Prieto J, Valenzuela-Santibáñez V. Model of socio-cultural dimensions involved in adherence to antiretroviral therapy for HIV/AIDS in public health care centers in Chile. AIDS Care. 2016;28(11):1441-7.

25. Lyimo RA, Stutterheim SE, Hospers HJ, T de Glee, A van der Ven, $M$ de Bruin. Stigma, Dsiclousee, Coping, and Medication Adherence among people living with HIV/AIDS in Northern Tanzania. AIDS PATIENT CARE and STD. 2014;28(2):98-105.

26. Turan B, Smith W, Cohen MH, Wilson TE, Adimora AA, Merenstein D, et al. Mechanisms for the Negative Effects of Internalized HIV-Related Stigma on Antiretroviral Therapy Adherence in Women: The Mediating Roles of Social Isolation and Depression. J Acquir Immune Defic Syndr. 2016;72(2):198205.

27. McCoy K, Higgins M, Zuñiga JA, M McDonnell Holstad. Age, Stigma, Adherence and Clinical Indicators in HIV-Infected Women. HIV/AIDS Res Treat. 2015;(SE3):S1-S8.

28. Li L, Lin C, Ji G. Gendered Aspects of Perceived and Internalized HIVRelated Stigma in China. Women \& Health. 2016; [Epub ahead of print].

29. Valencia-Garcia D, Rao D, Strick L, Simoni JM. Women's experiences with HIV-related stigma from health care providers in Lima, Peru: "I would rather die than go back for care". Health Care Women Int. 2016; [Epub ahead of print].

30. Rivera-Diaz M, Varas-Dias N, ReyesEstrada M, Suro B, Coriano D. Factores socio-estructurales y el estigma hacia el VIH/SIDA: experiencias de puertorriqueños/as con VIH/SIDA al acceder servicios de salud. Salud Soc. 2012;3(2):180-92.

Correspondencia: Jessica Hanae Zafra Tanaka

Dirección: Av. Conquistadores 365 - San Isidro

Teléfono: 994371991

Correo electrónico: j.zafra.t@gmail.com 